\title{
Avaliação da função autonômica cardiovascular em portadores de lesão medular submetidos à variabilidade da frequência cardíaca
}

\author{
Assessment of cardiovascular autonomic function in patients with spinal \\ cord injury submitted to heart rate variability
}

\author{
J.B. Caldeira, A.G. Sancho, F. Manoel, J.S. Rosa
}

ARTIGO ORIGINAL | ORIGINAL ARTICLE

\begin{abstract}
O objetivo foi avaliar as repercussões cardiovasculares do sistema nervoso autônomo através da avaliação da variabilidade da frequência cardíaca (VFC) em indivíduos portadores de lesão medular completa em diferentes níveis. Participaram do estudo 32 indivíduos, divididos em 3grupos: 13 pacientes tetraplégicos (G1), 8 pacientes paraplégicos (G2) e 11 indivíduos saudáveis formando o grupo controle (GC). Todos foram submetidos a avaliação VFC, realizada a partir dos dados obtidos pelo registro de 15 minutos, gerados pelo monitor cardíaco, interligado a um computador portátil, que registrava em gravação on-line os intervalos RR. A FC de repouso em posição supina no G1 foi menor quando comparada ao G2 e GC. A VFC foi maior no G1 na posição supina. A VFC foi menor na posição ortostática no G1 em comparação ao G2 e GC, como consequência da maior queda da pressão arterial. A análise espectral mostrou no G1 e GC redução da potência de alta frequência (diminuição da atividade vagal) e aumento da relação B/A representando a intensa diminuição do tônus vagal no G1 e aumento da atividade adrenérgica no GC. Os resultados evidenciaram uma disfunção autonômica em tetraplégicos e resultados em paraplégicos equivalentes ao grupo controle.

Palavras-chave: lesão medular, função autonômica, reabilitação, atividade motora
\end{abstract}

ABSTRACT

The objective was to assess the effects of cardiovascular autonomic nervous system through the heart rate variability (HRV) in subject with complete spinal cord injury (SCI) at different levels. 32 subjects participated in the study, divided into three groups: 13 tetraplegic patients (G1), 8 paraplegic patients (G2) and 11 healthy subjects formed the control group (CG). All underwent the assessment HRV, made from data obtained by record of 15 minutes, generated by the cardiac monitor, connected to a laptop, wich recorded on recording on-line RR intervals. The HR at rest in the supine position was lower in G1 compared to G2 and CG. HRV was highest in G1 in the supine position. HRV was lower in the standing position in the G1 compared to G2 and CG, as a consequence of the greater decrease in BP. Spectral analysis showed a reduction in G1 and GC of high-frequency power (decrease in vagal activity) and increased the ratio B / A represents the intensity decreased vagal tone in G1 and increased adrenergic activity in GC. The results showed an autonomic dysfunction in tetraplegics and paraplegics equivalent results in the control group.

Keywords: spinal cord injury, autonomic function, rehabilitation, motor activity

Submetido: 30.03 .2012 | Aceite: 29.01.2013

Jefferson Braga Caldeira, Alexandre Gomes Sancho, Felismar Manoel, João Luiz da Silva Rosa. Universidade UNIGRANRIO, Brasil.

Endereço para correspondência: Prof. Ms. Jefferson Braga Caldeira, Curso de Fisioterapia da UNIGRANRIO. Av. Prof. José de Souza Herdy, n 1.160, Bairro 25 de agosto, Duque de Caxias, CEP 25071-202, RJ, Brasil.

E-mail: jbraga@unigranrio.com.br 
O traumatismo da coluna vertebral pode lesar de maneira irreversível a medula espinhal e suas raízes nervosas, produzindo assim, alterações no circuito neural autonômico com consequências na função cardiovascular que estão frequentemente associadas a inúmeras condições clínicas, tendo muitas delas implicações na qualidade de vida e caráter prognóstico (Caldeira, Soares, \& Amorim, 2009; Wecht, De Meersman, Weir, Spungen, \& Bauman, 2003).

A disfunção autonômica que quase sempre se manifesta após a lesão medular, pode aparecer de forma aguda ou crônica, ser reversível ou não e corresponder a disfunção de um ou dos dois ramos do sistema nervoso autônomo (SNA). Quando estão presentes, trazem consigo manifestação clínicas, como: hipotensão ortostática, taquicardia de repouso, infarto do miocárdio sem dor, parada cardiorrespiratória, entre outras. Desta maneira a caracterização, a detecção precoce e a prevenção de episódios de disautonomias constituem fatores extremamente relevantes ao tratamento de portadores de lesão medular (Guyton \& Hall, 2006).

Encontramos descritos na literatura, vários testes com o objetivo de avaliar o sistema nervoso autônomo bem como suas repercussões cardiovasculares. O estudo da variabilidade da frequência cardíaca (VFC) é um método que nos permite analisar as flutuações que ocorrem durante períodos curtos ou prolongados da frequência cardíaca (FC) (Kleiger, Bosner, Rottman, \& Stein, 1993; Malik \& Camm, 1995) tendo a vantagem de possibilitar uma avaliação seletiva e não invasiva da função autonômica (Alonso et al., 1998; Ditor et al., 2005; Reis, Moreno, Sakabe, Catai \& Silva, 2005; Lopes et al., 2007; Vanderlei, Pastre, Hoshi, Carvalho, \& Godoy, 2009). As medidas do domínio do tempo são índices obtidos de um registro contínuo de eletrocardiograma (ECG) a partir do qual se determina a dispersão da duração dos intervalos entre o complexo QRS normais, isto é, resultantes de despolarização sinusal.
A VFC pode ser também avaliada através das medidas no domínio de frequência (Malliane, Pagani, Lombardi, \& Cerruti, 1991; Mortara, Rovere, \& Pantaleo, 1994; Malik \& Camm, 1995; Aubert, Seps, Beckers, 2003; Vanderlei et al., 2009), sendo estas medidas derivadas da análise espectral que decompõe a variabilidade total da FC em seus componentes causadores, apresentando-os segundo a frequência com que alteram a FC. O cálculo da densidade espectral pode ser obtido através dos algorítmicos que utilizam Transformadas Rápidas de Fourier ou modelos auto-regressivos. Resultados obtidos por estes métodos demonstram que em humanos o espectro de potência da variabilidade fisiológica apresenta normalmente quatro faixas de frequência: alta frequência (AF: 0,15 a 0,40 Hz); baixa frequência (BF: 0,04 a 0,15 Hz) (Furlan, Gussetti, \& Crivellaro, 1990; Tsuji, Larson, \& Venditti, 1996; Aubert et al., 2003; Novais, Sakabe, Takahashi, Gongora, Taciro, \& Martins, 2004; Godoy, Takakura, \& Correa, 2005). Tem sido correlacionada ao sistema barorreceptor e termorregulador à atividade periférica vasomotora e ao sistema renina-angiotensina (Kleiger et al., 1993; Mortara et al., 1994); muito baixa frequência (MBF; 0,01 à $0,04 \mathrm{~Hz}$ ) (Kleiger et al., 1993); ultra baixa frequência (UBF: $10-5$ à $10-2 \mathrm{~Hz}$ ).

As bandas $\mathrm{AF}$ e $\mathrm{BF}$ e suas potências aparecem para fornecer uma importante taxa para avaliar o complexo de interação entre as atividades reguladoras neurais e algumas variabilidades cardiovasculares. Estudos recentes mostraram que o aumento da atividade simpática, estava relacionado com o aumento do componente de baixa frequência, ao passo que a acentuada atividade vagal está acompanhada com relativo aumento do componente de alta frequência. Em resumo, as potências desses ritmos aparecem para demonstrar uma relação recíproca como a comumente encontrada no índice simpatovagal. Por outro lado, baixa frequência e pressão arterial sistólica parecem ser marcas fidedignas da modulação simpática e 
atividade motora (Malliane et al., 1991).

A evolução dos efeitos da desconexão simpática em lesados medulares ao longo do tempo, não são ainda bem conhecidas. Além disso, é provável que a gravidade desses efeitos dependa da natureza e o nível da lesão, de outros fatores associados às atividades diárias mantidas pelo portador da lesão. Finalmente, os prováveis efeitos benéficos do tratamento fisioterapêutico sobre a evolução das disreflexias autonômicas decorrentes da lesão medular ainda permanecem em grande parte por esclarecer.

Portanto, este trabalho teve como objetivo avaliar as repercussões cardiovasculares do sistema nervoso autônomo através da avaliação da variabilidade da frequência cardíaca (VFC) em indivíduos portadores de lesão medular completa em diferentes níveis.

\section{MÉTODO}

\section{Amostra}

Estudo experimental que após diagnóstico clínico-neurológico, foram selecionados na clínica de fisioterapia do Centro Universitário do Triângulo (UNITRI), Associação Fluminense de Reabilitação (AFR) e Associação Niteroiense de Deficientes Físicos (ANDEF), 32 indivíduos, divididos em 3 grupos, no qual: o primeiro grupo (G1), formado por 13 pacientes tetraplégicos com idade média de 34.53 anos \pm 11.26 , que sofreram lesão medular completa alta (acima de C7), sendo três do sexo feminino e 10 do sexo masculino. O segundo grupo (G2), formado por 8 pacientes paraplégicos com idade média de 33.37 anos \pm EP 4.15, sendo 7 do sexo masculino e 1 do sexo feminino. O terceiro grupo ou grupo controle (GC), formado por 11 indivíduos saudáveis com idade média de 27.9 anos \pm EP 2.27, sendo 7 do sexo masculino e 4 do sexo feminino. Todos os pacientes estavam normotensos, normocárdicos e afebris há pelo menos um mês e sem qualquer sinal ou sintoma de infecção, inclusive urinária.

\section{Instrumentos e Procedimentos}

Foi adaptado ao tórax do participante, um monitor cardíaco (Polar Vantage) que através de seu transmissor interligado a um computador portátil (Pentium 100), que por sua vez, registrava em gravação on-line os intervalos $\mathrm{RR}$ em tempo real do ciclo cardíaco, para avaliação da variabilidade da frequência cardíaca (VFC). Os monitores de frequência cardíaca Polar promovem informações em tempo real do ritmo cardíaco, registrando os intervalos $\mathrm{RR}$, que são definidos como a duração do tempo entre complexos QRS adjacentes, isto é, a extensão do ciclo cardíaco. O instrumento detecta as ondas $\mathrm{R}$ do eletrocardiograma com uma frequência de $500 \mathrm{~Hz}$ e resolução temporal de $1 \mathrm{~ms}$ (Ruha, Sallinen, \& Nissilä, 1997; Vanderlei et al., 2009).

O cinto transmissor foi posicionado de forma justa o suficiente, para não permitir o movimento dos eletrodos durante os testes. A transmissão de informações é realizada através de radiofrequência e pode sofrer influências de campos eletromagnéticos externos, portanto, foram excluídas todas as possíveis fontes perturbadoras dos sinais como: telefones celulares, antenas de rádio, motores elétricos e até mesmo monitores de vídeos. Para melhor captação dos sinais do Polar, a interface receptora foi mantida a uma distância entre $30 \mathrm{~cm}$ a $60 \mathrm{~cm}$ do cinto receptor.

Os pacientes repousaram em uma cama tipo ortostática, por um período não inferior a três minutos; em seguida eles foram orientados a respirar, ouvindo uma fita cassete que continha instruções de comando do ciclo respiratório "inspire e expire" com uma frequência respiratória normal e fisiológica de 15 ciclos por minuto. O controle da gravação da frequência respiratória foi conduzido por marcação da inspiração e expiração, com auxílio de um metrômetro.

A gravação de 15 minutos foi realizada em duas posições: primeiro na posição de decúbito dorsal (horizontal) e após cinco minutos, na 
posição ortostática com inclinação da cama a uma posição de 70 graus. As informações foram interpretadas pelo programa Polar Precision Performance TM Software for Windows Version 2.0. Em uma gravação de 15 minutos do sinal eletrocardiográfico contínuo, cada onda $\mathrm{R}$ foi detectada e os intervalos entre os batimentos considerados normais foram computados para a construção da série temporal.

Para avaliar a VFC no domínio do tempo foram determinados todos os complexos QRS e o seu suposto intervalo RR normal. Então, todos os intervalos entre os complexos de QRS adjacentes constituíram uma determinação da FC. O programa de filtragem foi elaborado em planilha de cálculo (Microsoft Excel ${ }^{\circledR}$ ) excluindo-se os dados que excederam a $20 \% \mathrm{em}$ valor absoluto, do batimento adjacente. Desta forma, foram separados os batimentos normais dos artefatos e ectopias, sendo montada uma série temporal somente de intervalos $R R$ normais. Após esta análise, foram realizados os estudos da variabilidade da frequência cardíaca no domínio do tempo: SDNN, SDNNi, SDANN, RMSSD, pNN50 e RR médio.

Para o domínio da frequência a partir das séries temporais dos intervalos $\mathrm{RR}$, foram localizados os intervalos cujo valor diferenciasse em mais de $20 \%$ do intervalo anterior (Malik \& Camm, 1996). Após esta detecção automática, foram determinados subjetivamente, os intervalos e pontos considerados anormais ou trechos classificados pelo observador como contendo ruído.

Para a realização dos cálculos foi utilizado o programa MATLAB - MATHWORKS, através deste foram mantidas as bases dos tempos originais, e realizadas interpolações por "cubic spline" (polinômio de terceiro grau) a uma frequência de amostragem de $10 \mathrm{~Hz}$. O sinal obtido foi dividido em oito trechos de tamanho aproximado para uma potência de dois e calculado o espectro de potência através da Transformada Rápida de Fourier para cada trecho. Os parâmetros derivados do espectro de potência foram: a potência total, as potências de baixa $(0.08 \mathrm{a} 0.15 \mathrm{~Hz})$ e alta frequência $(0.15 \mathrm{a} 0.40 \mathrm{~Hz})$ e o índice baixa frequência/alta frequência (B/A).

Como critérios de exclusão foram considerados pacientes que apresentavam diabetes, infecção urinária, doença pulmonar obstrutiva crônica (DPOC), outras neuropatias autonômicas, lesão neurológica incompleta, instabilidade clínica, doença cardíaca prévia, uso de drogas neurolépticas.

$O$ presente trabalho atende às normas para a realização de pesquisa em seres humanos, conforme declaração de Helsinki de 1964 e a resolução 196/96 do Conselho Nacional de Saúde do Ministério da Saúde. Todos os participantes do estudo assinaram o Termo de Consentimento Livre e Esclarecido. O estudo teve seu projeto de pesquisa aprovado pela Comissão de Ética em Pesquisas da Clínica Escola de Fisioterapia da Universidade do Grande Rio (UNIGRANRIO), aprovado no dia 02/09/2008 e registrado sob o número 031/2008.

\section{Análise Estatística}

Os dados foram submetidos à uma análise univariada, através do teste de Kolmogorov-Smirnov para verificação da distribuição normal.

Para variáveis com mais de três grupos, utilizamos ANOVA simples; para variáveis com duas situações e três grupos, utilizamos ANOVA de dois fatores para medidas repetidas. Quando foram obtidos fatores de F significativos, foram realizadas comparações "posthoc” através do teste $t$ com correção de Bonferroni para comparações par a par.

Os resultados foram apresentados em forma de média e desvio-padrão, e valores de $p<.05$ foram considerados estatisticamente significantes.

\section{RESULTADOS}

\section{Variabilidade da Frequência Cardíaca no Domínio do Tempo}

A média dos intervalos RR nos três grupos foi diferente quando comparados os valores 
obtidos na posição supina e aqueles obtidos na posição ortostática $(960 \pm 30.57 ; 902 \pm 27.13$; $862 \pm 36.05$ vs $756 \pm 32.50 ; 850 \pm 47.43 ; 681$ \pm 96.76 ) respectivamente para os grupos dos tetraplégicos, paraplégicos e controle. Observa-se também que em comparação com a mesma posição, os valores do grupo dos tetraplégicos foram significantemente maiores nas posições supina em comparação com os grupos dos paraplégicos e controle $(960 \pm 30.57$ vs $862 \pm$ 36.05 ; $850 \pm 47.43$ vs $681 \pm 96.76)$ respectivamente (Figura 1). Durante o ortostatismo os valores de RR médio do grupo dos paraplégicos foram maiores que os demais.

A análise da variabilidade total, calculada como o desvio-padrão, demonstrou que os valores do grupo dos tetraplégicos na posição supina é maior do que os valores dos grupos dos paraplégicos e controle (Figura 2). Todavia na posição ortostática a variabilidade do grupo dos tetraplégicos é menor que os grupos dos paraplégicos e controle. Finalmente comparando-se para cada grupo os valores na posição supina e ortostática, observa-se que no grupo dos tetraplégicos e controle ocorre diminuição da variabilidade na posição supina, enquanto no grupo dos paraplégicos não houve diferença entre estes valores.

A média dos desvios-padrão dos intervalos RR normais calculados em intervalos de 5 minutos, quando comparados com à sua posição supina foi menor em tetraplégicos e controle ( $51 \pm 9.03$ vs $24 \pm 2.57$ e $52 \pm 3.96$ vs $44 \pm 6.63$ ) (Figura 3). Quando todos os grupos são comparados na mesma posição observa-se que o valor do grupo dos tetraplégicos na posição ortostática é significantemente menor que dos paraplégicos e controle $(24 \pm 2.57$ vs $50 \pm 5.99$ e $44 \pm 6.63$ ) respectivamente.

O desvio-padrão das médias dos intervalos RR normais calculados em intervalos de 5 minutos foi maior em tetraplégicos, quando comparados com paraplégicos na posição supina (32.66 \pm 6.82 vs $20.00 \pm 5.51)$ (Figura 4).

Quando os valores da raiz quadrada das diferenças sucessivas entre os intervalos $R R$ normais adjacentes ao quadrado são analisados (Figura 5), os valores obtidos pelo grupo dos tetraplégicos e controle foram menores quando comparados com a sua posição supina (54.35 \pm 11.45 vs $18.89 \pm 2.44$ e $39.95 \pm 3.79$ vs 31.84 \pm 5.05) respectivamente. Quando comparados com a mesma posição, os valores do grupo dos paraplégicos foi maior que os dos tetraplégicos e controle $(39.05 \pm 4.06$ vs $18.89 \pm 2.44$ e $31.84 \pm 5.05)$ respectivamente.

$O$ percentual de intervalos $R R$ normais que diferiu mais que 50 milessegundos de seu adjacente na posição supina é semelhante em todos os grupos (Figura 6). Na passagem para

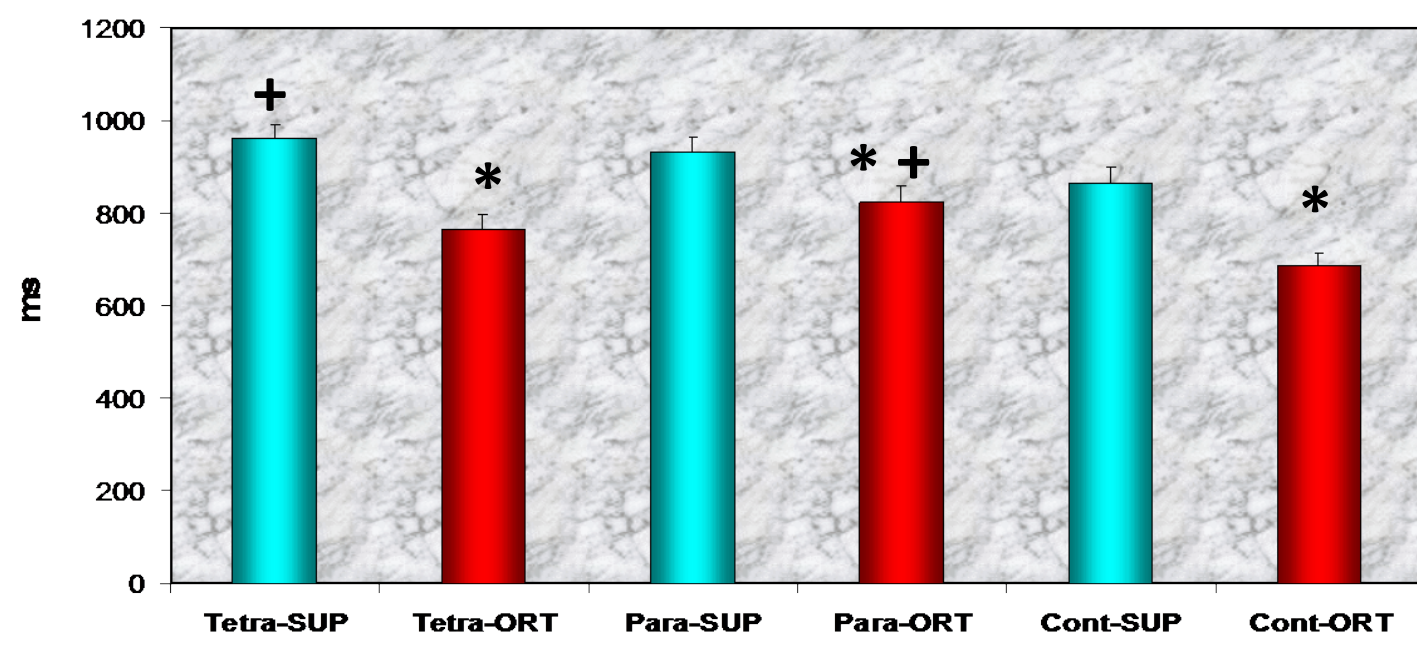

Figura 1. ${ }^{*} p<.05$ vs posição supina no mesmo grupo; $+p<.05$ vs controle na mesma posição. 


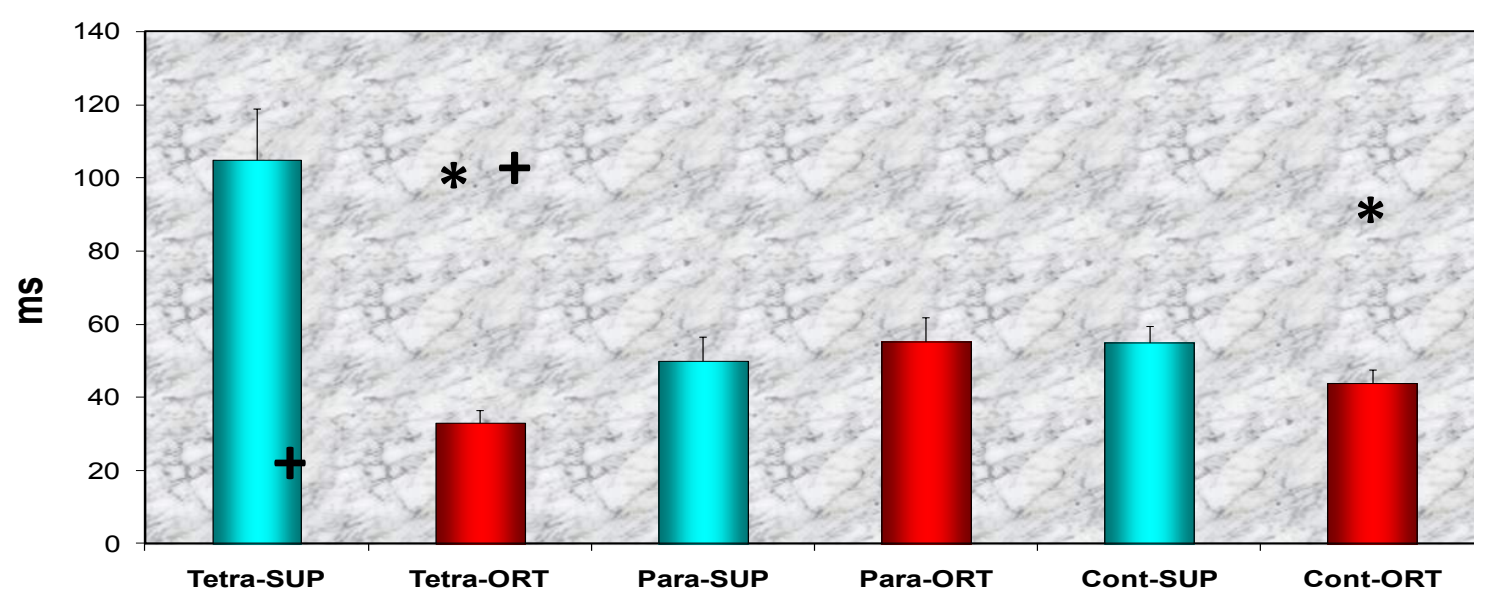

Figura $2 .{ }^{*} p<.05$ vs supina no mesmo grupo; $+p<.05$ vs paraplégicos e controle na mesma posição.

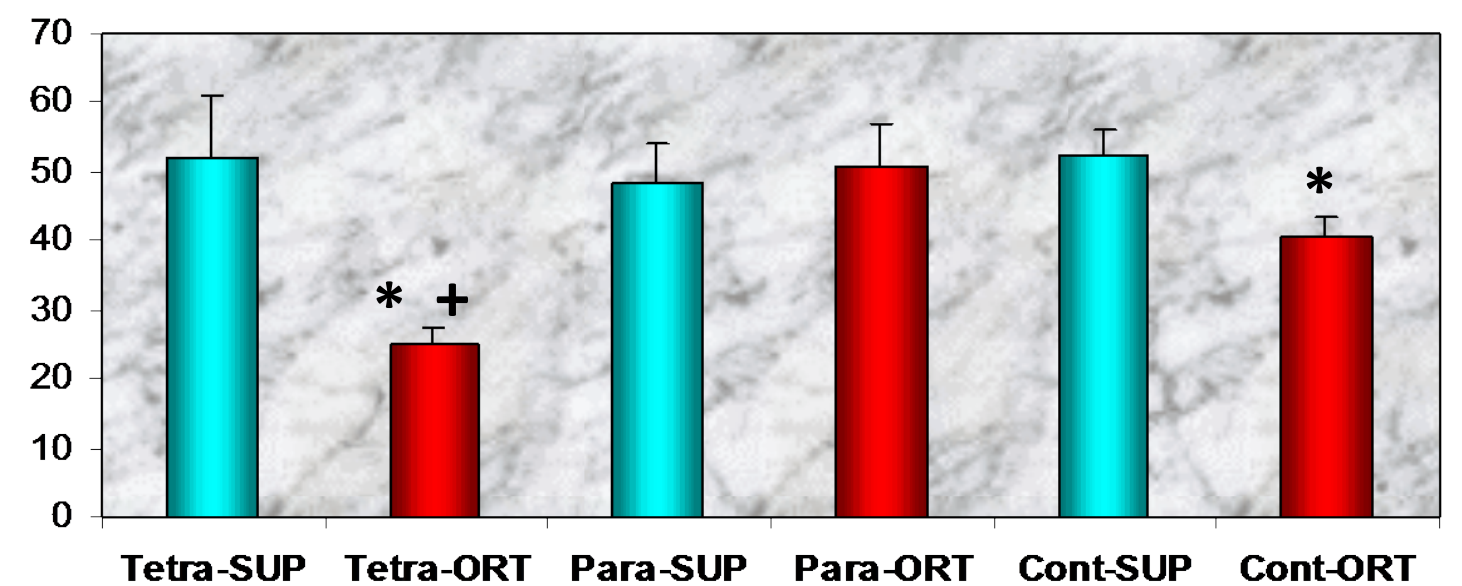

Figura $3 .{ }^{*} p<.05$ vs supina no mesmo grupo; $+p<.05$ vs paraplégicos e controle na mesma posição.

a posição ortostática, observa-se diminuição significativa nos grupos dos tetraplégicos e controle, sem variação significativa no grupo dos paraplégicos. Consequentemente o índice pNN50 na posição ortostática é maior no grupo dos paraplégicos que os demais.

\section{Variabilidade da Frequência Cardíaca no Domínio da Frequência}

A potência total do espectro da variabilidade da frequência cardíaca estava presente dentro dos parâmetros de normalidade nos três grupos avaliados na posição supina (40418 \pm 15144; $37724 \pm 9543 ; 46691 \pm 6538$; respectivamente). Quando os indivíduos foram colocados em posição ortostática observa-se que a potência total diminuiu significativamente nos grupos dos tetraplégicos e controle em comparação com a sua posição supina (10330 \pm 1991, $42614 \pm 9683)$ respectivamente, não se observando esta diminuição no grupo dos paraplégicos (Figura 7). Consequentemente na posição supina os valores da potência total do espectro são maiores para os grupos dos paraplégicos quando comparados aos grupos dos tetraplégicos e controle (10330 $\pm 1991 ; 43752$ \pm 10476; $42614 \pm$ 9683; respectivamente).

A média da potência associada à faixa de baixa frequência quando comparados na mesma posição, mostrou-se diminuída nas duas posições no grupo dos tetraplégicos em relação aos outros grupos $(0.09 \pm 0.05$ vs $0.171 \pm 0.02$; $0.15 \pm 0.01$ e $0.11 \pm 0.05$ vs $0.18 \pm 0.07 ; 0.16$ \pm 0.09 ) respectivamente (Figura 8 ).

Quando foram posicionados na posição de ortostatismo, a média da potência de baixa 


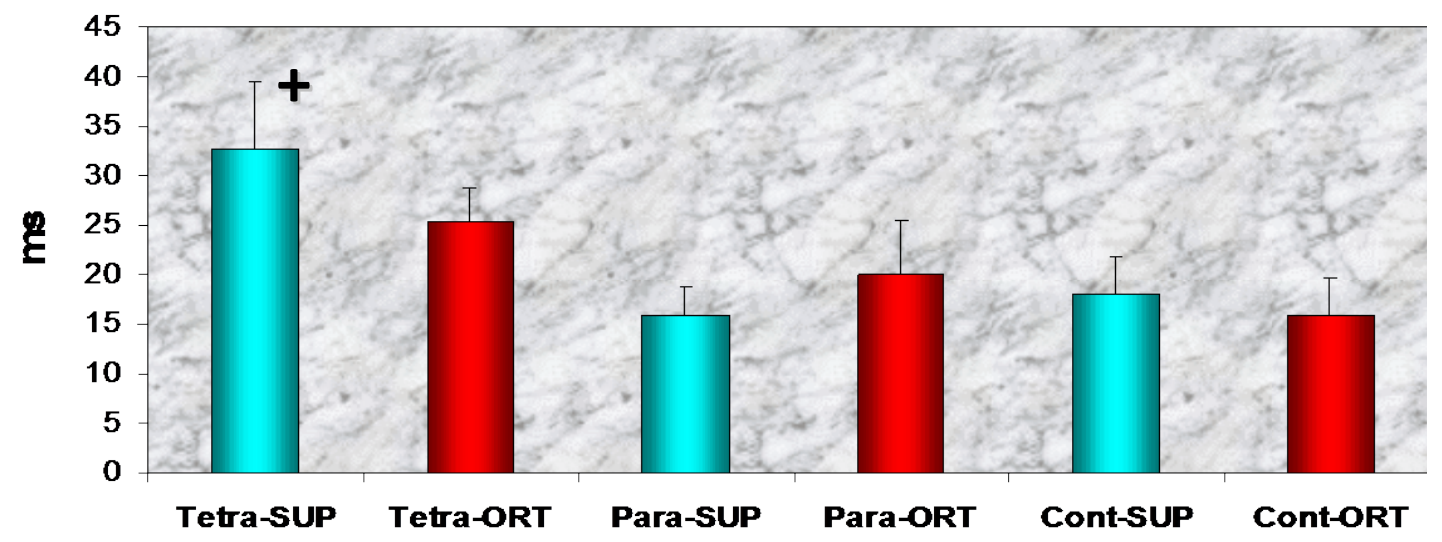

Figura $4 .+p<.05$ vs paraplégicos na mesma posição.

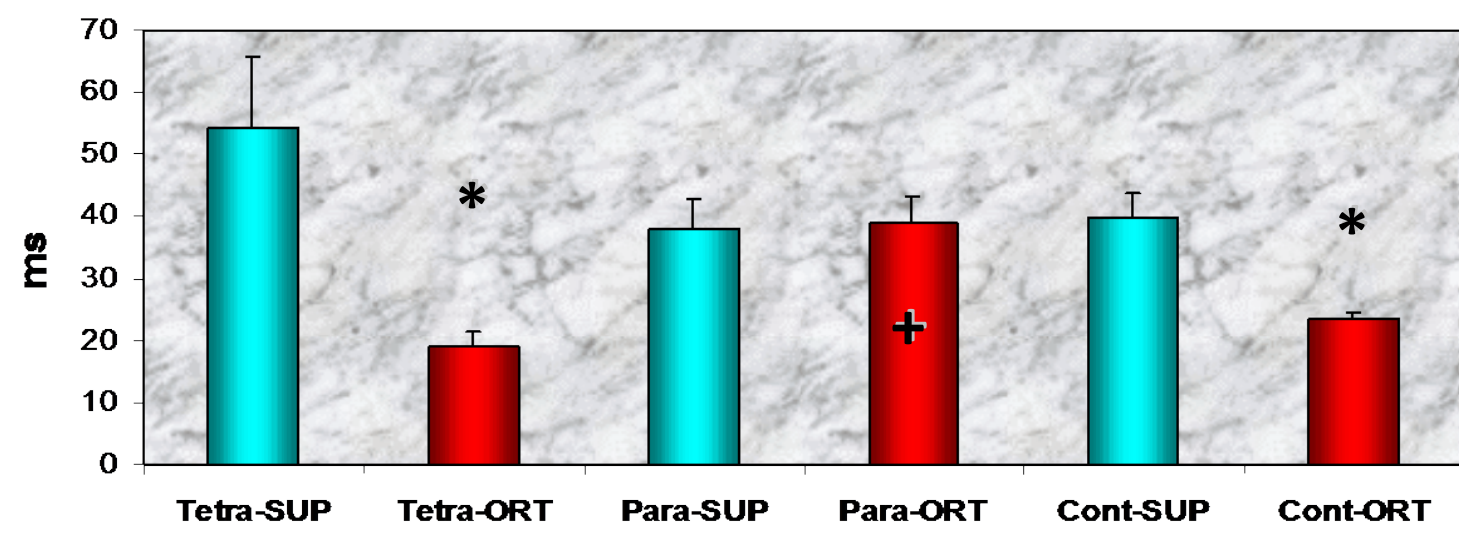

Figura 5. ${ }^{*} p<.05$ vs supina no mesmo grupo; $+p<.05$ vs tetraplégicos e controle na mesma posição.

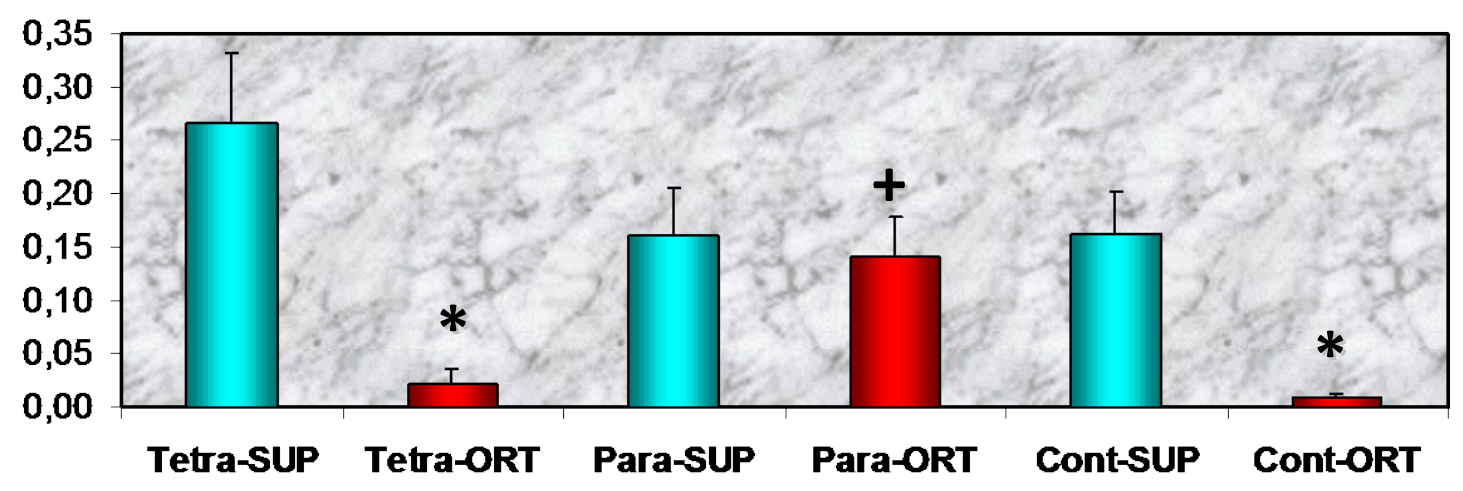

Figura 6.

frequência subiu significantemente no grupo controle em relação à sua posição supina $(0.16$ \pm 0.09 vs $0.15 \pm 0.05)$ respectivamente, sem variação significativa nos grupos dos tetraplégicos e paraplégicos (gráfico 8 ). Observou-se que a média da potência associada à alta frequência foi menor em paraplégicos quando comparados com tetraplégicos e controle na posição supina $(0.16 \pm 0.02$ vs $0.27 \pm 0.0$; e $0.23 \pm 0.02$ ) respectivamente (Figura 9). Quando foram posicionados em posição ortostática a média da potência associada à alta frequência caiu nos três grupos tornando-se estatisticamente significante em tetraplégicos e controle, quando comparados com relação à suas posições supina $(0.27 \pm 0.00$ vs $0.10 \pm 0.00$; e $0.23 \pm 0.02$ vs $0.13 \pm 0.02)$ respectivamente.

A média da relação $\mathrm{B} / \mathrm{A}$ foi menor no grupo 


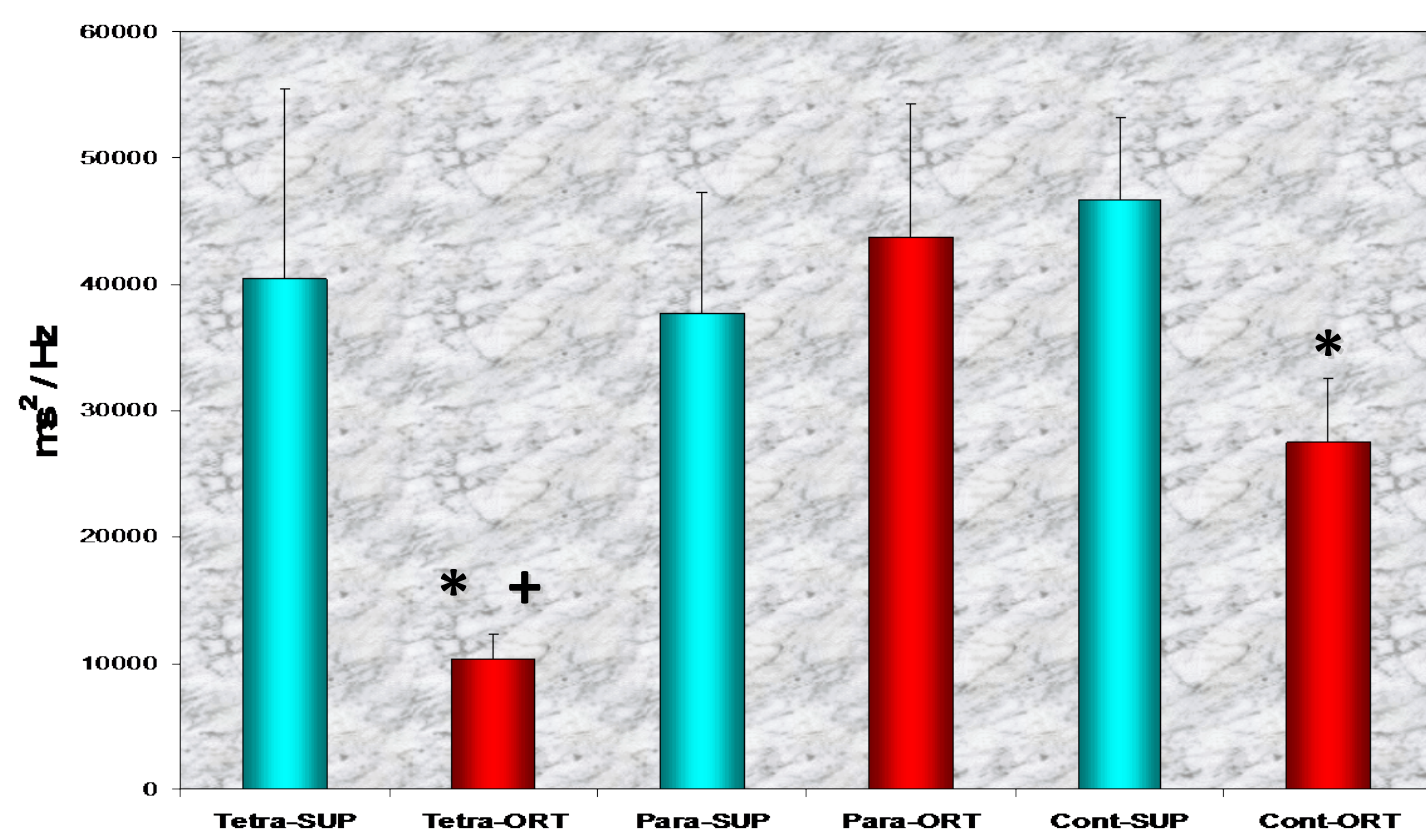

Figura 7. ${ }^{*} p<.05$ vs supina na mesmo grupo; $+p<.05$ vs paraplégicos e controle na mesma posição

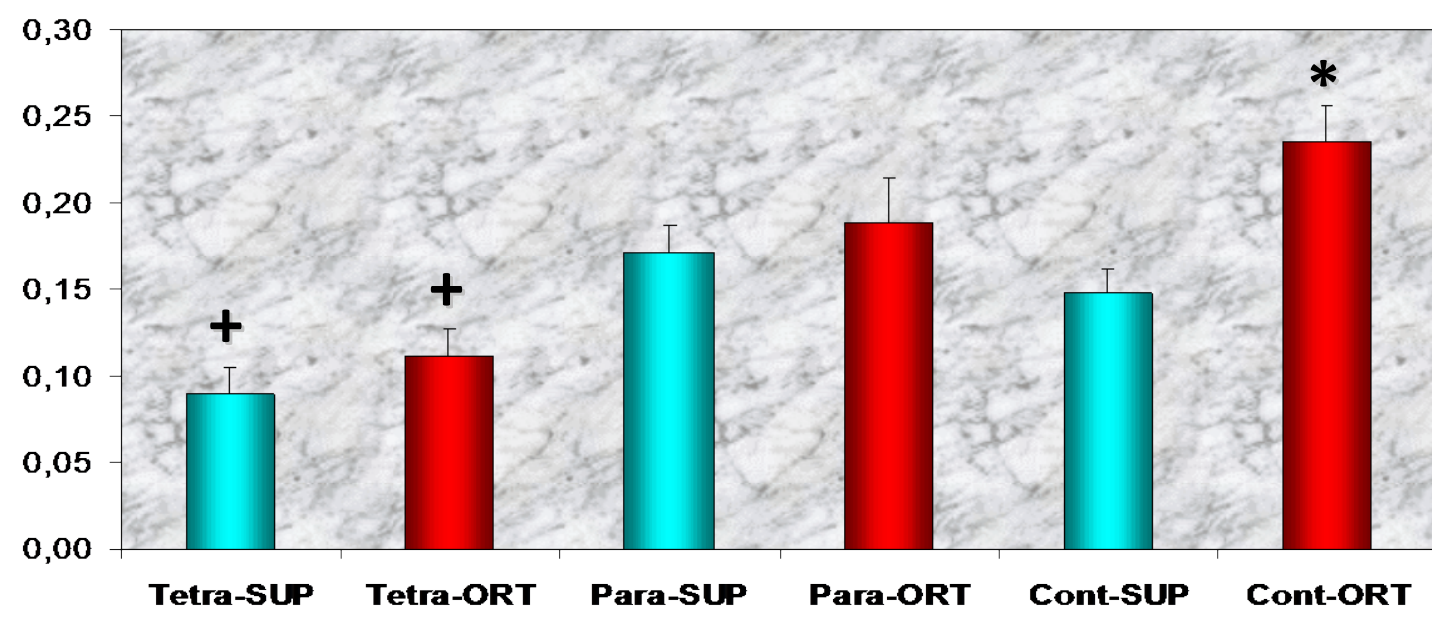

Figura $8 .{ }^{*} p<.05$ vs supina no mesmo grupo; $+p<.05$ vs paraplégicos e controle na mesma posição.

dos tetraplégicos em comparação com paraplégicos e controle na posição supina $(0.51 \pm$ $0 ; 1.24 \pm 0.21 ; 0.76 \pm 0.13)$. Quando foram adaptados à posição ortostática, os valores da relação aumentaram nos três grupos, porém tornando-se significativos em tetraplégicos e controle $(0.51 \pm 0,00$ vs $2.97 \pm 1.00 ; 0.76 \pm$ 0.13 vs $1.54 \pm 0.41$ ) (Figura 10).

\section{DISCUSSÃo}

Uma transecção medular cervical pode produzir alterações no circuito neural autonômico com consequências na função cardiovascular que estão frequentemente associadas à inúmeras condições clínicas, tendo muitas delas implicações na qualidade de vida e caráter prognóstico (Ditor et al., 2005). Assim, a aferição ou qualificação do estado autonômico cardiovascular individual tem direta importância clínica, podendo ser útil para a definição de diferentes condutas médicas.

A diferença esperada entre tetraplégicos e paraplégicos em relação ao controle cardiovascular baseia-se na organização funcional do sistema nervoso autônomo. O SNA controla as funções viscerais coordenados por centros localizados no tronco cerebral e no hipotálamo, compondo-se de duas divisões ou 


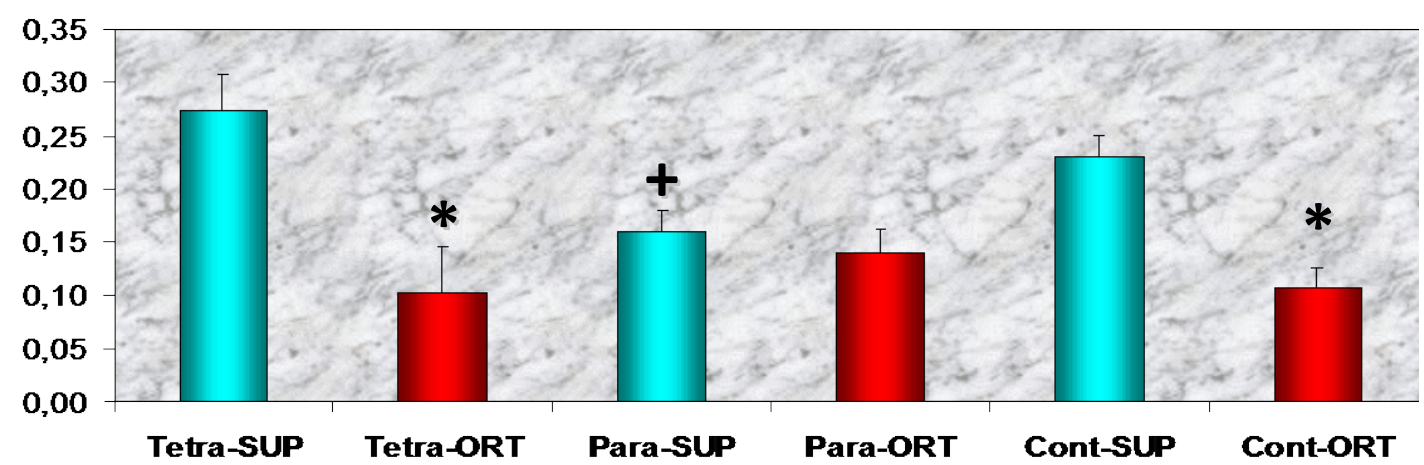

Figura 9. ${ }^{*} p<.05$ vs supina no mesmo grupo; $+p<.05$ vs tetraplégicos e controle na mesma posição.

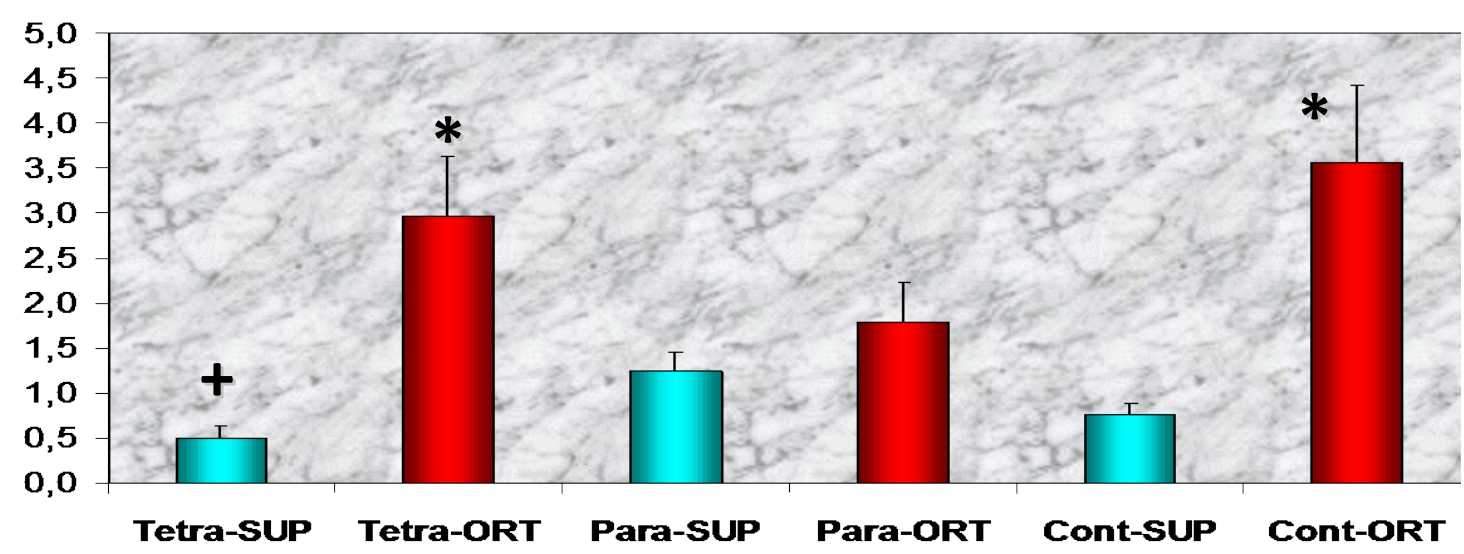

Figura $10{ }^{*} p<.05$ vs supina no mesmo grupo; $+p<.05$ vs paraplégicos na mesma posição.

ramos chamados de simpático e parassimpático. As fibras eferentes do sistema simpático ou adrenérgico emergem da medula dos segmentos torácicos e lombares inervando o coração e vasos sanguíneos. Desta forma, participa no aumento da atividade global do coração (aumento do inotropismo e do cronotropismo) e no controle da resistência vascular periférica. A inervação adrenérgica do coração se organiza em forma de plexo formado principalmente por fibras dos segmentos medulares $\mathrm{T} 1$ à $\mathrm{T} 3$, sendo que a parte superficial do plexo cardíaco situa-se junto ao arco aórtico, um tanto sobre o lado esquerdo entre este e a artéria pulmonar e a parte profunda do plexo, situa-se profundamente ao arco aórtico, entre a bifurcação da traquéia e cranialmente a artéria pulmonar (Kleiger et al., 1993). Por outro lado, o ramo parassimpático ou colinérgico, emerge do sistema nervoso central do tronco cerebral através de certos nervos cranianos e dos segmentos sacrais da medula.
Em relação ao sistema cardiovascular, o ramo parassimpático participa fundamentalmente no controle batimento-à-batimento da frequência cardíaca através do nervo vago (Malik \& Camm, 1996). Portanto, nos indivíduos paraplégicos, o nível de lesão medular está abaixo da inervação adrenérgica cardíaca permitindo seu controle pelos centros integradores no encéfalo. Em contraste, quando existe uma transecção medular acima dos segmentos torácicos, como nos tetraplégicos, ocorre uma desconexão do encéfalo com os neurônios motores simpáticos e as respostas reflexas de alteração de contratilidade miocárdica e vasoconstricção estariam ausentes, deixando íntegro apenas o controle parassimpático do coração.

\section{VFC no Domínio do Tempo}

As medidas do domínio do tempo são índices obtidos de um registro contínuo de eletrocardiograma (ECG) a partir do qual se determina a dispersão da duração dos intervalos entre o 
complexo QRS normais, isto é, resultantes de despolarização sinusal (Tabela 1).

Tabela 1.

Definição dos índices do domínio do tempo da variabilidade da frequência cardíaca

\begin{tabular}{ll}
\hline \hline Índices* Unidade & Definição \\
\hline
\end{tabular}

RR médio ms Media de todos os intervalos RR

SDNN ms Desvio-padrão de todos os intervalos RR normais

\begin{tabular}{|c|c|c|}
\hline SDNNi & $\mathrm{ms}$ & $\begin{array}{c}\text { Media dos desvios-padrão } \\
\text { dos intervalos RR normais } \\
\text { calculados em intervalos de } 5 \\
\text { minutos }\end{array}$ \\
\hline SDANN & $\mathrm{ms}$ & $\begin{array}{c}\text { Desvio-padrão das médias } \\
\text { dos intervalos RR normais } \\
\text { calculados em intervalos de } 5 \\
\text { minutos }\end{array}$ \\
\hline RMSSD & $\mathrm{ms}$ & $\begin{array}{c}\text { Raiz quadrada das diferenças } \\
\text { sucessivas entre intervalos RR } \\
\text { normais adjacentes ao quadrado }\end{array}$ \\
\hline pNN50 & $\%$ & $\begin{array}{l}\text { Percentual de intervalos RR } \\
\text { normais que diferem mais que } \\
50 \text { milessegundos de seu adja- } \\
\text { cente }\end{array}$ \\
\hline
\end{tabular}

* As siglas dos índices referem-se às abreviaturas dos termos em inglês seguindo padrão internacional (Malik \& Camm, 1995)

Na posição de repouso supino, observamos maior intervalo RR (bradicardia) no grupo dos tetraplégicos em comparação com os paraplégicos e controle $(960 \pm 30$ vs $902 \pm 27$ e $862 \pm 36 ; p<.05$, respectivamente). Este achado discorda com os de Koh et al. (1994), que não encontraram diferença na duração dos intervalos RR entre tetraplégicos e indivíduos controle. Apesar da discordância, pode-se explicar de forma lógica nosso resultado do ponto-de-vista do controle autonômico cardiovascular. Segundo, Araújo, Nóbrega e Castro (1992), os valores de FC são determinados pela interação de três fatores: $1^{\circ}$ ) frequência de despolarização espontânea do nódulo sinusal (FC intrínseca); $2^{\circ}$ ) tônus adrenérgico e $3^{\circ}$ ) tônus vagal. Em repouso a FC intrínseca recebe maior influência do tônus vagal, mas a presença do tônus adrenérgico tem algum efeito taquicardizante. Por esta razão, nos indivíduos tetraplégicos, a diminuição ou ausência do tônus adrenérgico permite uma predominância ainda maior do tônus vagal, determinando um valor de FC menor do que em indivíduos que possuem tônus adrenérgico preservado, como nos paraplégicos e indivíduos controle.

O grupo dos tetraplégicos apresentou maior VFC na posição supina, medida pelos índices no domínio do tempo SDNN e SDANN. Este foi provavelmente um efeito secundário à menor FC, uma vez que existe uma relação inversa entre FC e VFC.

Como esperado, durante adoção da posição ortostática observou-se uma diminuição do intervalo RR (taquicardia) nos três grupos $(960 \pm 30$ vs $756 \pm 32 ; 902 \pm 27$ vs $850 \pm$ $47 ; 862 \pm 39$ vs $682 \pm 96 ; p<.05)$ respectivamente. Esta redução foi mais evidente em tetraplégicos. Concomitantemente, registramos uma diminuição da variabilidade no grupo dos tetraplégicos e controle (SDNN, SDNNi, RMSSD, pNN50) permanecendo inalterado no grupo dos paraplégicos. Apesar de não termos uma explicação clara para a não modificação dos índices no grupo dos paraplégicos, a diminuição da VFC nos tetraplégicos e controle reflete provavelmente a diminuição no tônus vagal cardíaco.

Quando comparados na posição ortostática, os tetraplégicos apresentaram menor VFC (SDNN e SDNNi) do que os grupos dos paraplégicos e controle, provavelmente refletindo a maior inibição vagal naquele grupo em consequência da maior queda da PA. Em outras palavras, a resposta baroreflexa neste grupo, que decorre apenas da inibição vagal, foi mais intensa devido à menor PA que se desenvolveu em posição ortostática pela a ausência do aumento do tônus adrenérgico.

\section{VFC no Domínio de Frequência}

As representações da modulação adre- 
nérgica e vagal da FC na análise espectral são extremamente complexas e controversas, principalmente o componente de Baixa frequência.

\section{Potência Total}

Os resultados da potência total mostram que a VFC diminuiu nos grupos tetraplégicos e controle em consequência à inibição vagal. Novamente, não foi possível encontrar uma explicação para a falta de resposta nos paraplégicos. Na posição ortostática, a potência total foi menor nos tetraplégicos refletindo uma grande inibição vagal devido à maior queda da PA.

\section{Baixa Frequência}

A potência de baixa frequência, que parece refletir tanto a atividade vagal quanto simpática, durante a adoção da posição ortostática, aumentou no grupo controle, mas não se alterou nos tetraplégicos e paraplégicos, confirmando os dados de Guzzetti et al. (1994), mas contradizem os de Cooke et al. (1999), que não detectaram modificações na potência de baixa frequência em indivíduos sadios quando em inclinação. Confirmando a complexidade do tema, Inoue, Miyake, Kumashiro, Ogata, e Yoshimura (1990) documentaram que flutuações de baixa frequência, estavam ausentes em indivíduos tetraplégicos durante posição ortostática. Este achado foi interpretado como uma intensa inibição vagal, considerando-se o espectro de baixa frequência como representativo tanto da atividade vagal quanto adrenérgica.

Observou-se que apesar de menor que os grupos controle e paraplégicos, a média do componente de baixa frequência em tetraplégicos estava dentro da faixa de normalidade $(0,09 \mathrm{~Hz})$ mesmo com dois dos 13 indivíduos apresentando resultados abaixo do esperado. Observamos também que em vários indivíduos o componente de baixa frequência mostrou-se nos limites inferiores da normalidade.

\section{Alta Frequência}

Em relação a alta frequência, parece existir certo consenso de que representa a modulação respiratória da FC mediada pela flutuação da atividade vagal cardíaca, pois os mesmos são coincidentes com os descritos por Reis, Bastos, Mesquita, Romeo, e Nóbrega (1997). Nossos dados mostraram que os grupos dos tetraplégicos e controle tiveram a potência de baixa frequência diminuída com a mudança de decúbito para 70 graus. Estes achados corroboram os de Guzzetti et al. (1994) e Koh, Brown, Beightol, Chang, e Eckberg (1994).

\section{Relação B/A}

A relação B/A é considerada por Reis et al. (1997), como um marcador da atividade simpática, uma vez que o componente de baixa frequência engloba atividade simpática e parassimpática e o componente de alta frequência representa apenas a modulação vagal. O grupo dos tetraplégicos se diferenciou dos demais quando comparados na mesma posição (0.51 \pm 0.00 vs $1.24 \pm 0.21 ; 0.76 \pm 0.13 ; p<.05$ respectivamente). Estes resultados confirmam a proposta de que a relação B/A representa a atividade adrenérgica. Quando colocados na posição ortostática, observamos que a relação B/A aumentou nos grupos tetraplégicos e controle $(0.51 \pm 0.00$ vs $1.54 \pm 1.00$; $0.76 \pm 0.13$ vs $1.54 \pm 0.41)$ respectivamente. $\mathrm{O}$ aumento da relação observado nos tetraplégicos pode ser atribuído à intensa inibição da atividade vagal, que é o denominador da relação, uma vez que o sistema nervoso simpático se encontra desprovido de conexão com o encéfalo. Nos indivíduos sadios este aumento decorreu mais provavelmente do aumento da atividade simpática.

\section{CONCLUSÕES}

A FC de repouso em posição supina nos tetraplégicos foi menor quando comparada aos paraplégicos e controles, refletindo a ausência do tônus adrenérgico nestes pacientes.

A VFC foi maior nos tetraplégicos na posição supina. A VFC foi menor na posição 
ortostática em tetraplégicos em comparação à paraplégicos e controle, como consequência da maior queda da PA.

A análise espectral mostrou em tetraplégicos e controle redução da potência de alta frequência (diminuição da atividade vagal) e aumento da relação $B / A$ representando a intensa diminuição do tônus vagal nos tetraplégicos e aumento da atividade adrenérgica nos controles.

\section{Agradecimentos:}

A todo o corpo discente da UNIGRANRIO que participaram diretamente ou indiretamente deste estudo.

\section{Conflito de Interesses:}

Nada declarado.

\section{Financiamento:}

Este estudo não foi financiado por qualquer fundação, entidade pública ou privada.

\section{REFERÊNCIAS}

Alonso, D. O, Forjaz, C. L. M., Rezende, L. O., Braga, A. M. F. W., Barretto, A. C. P., Negrão, C. E., Rondon, M. U. P. B. (1998). Comportamento da Frequência Cardíaca e da sua Variabilidade durante as diferentes Fases do Exercício Físico Progressivo Máximo. Arquivos Brasileiros de Cardiologia, 71(6),787-792.

Araújo, C. G .S., Nóbrega, A. C., \& Castro, C. L. B. (1992). Heart rate responses to deep breathing and 4-seconds of exercise before and after pharmacological blockade with atropine and propranolol. Clinical Autonomic Research, 2, 35-40.

Aubert, A. E., Seps, B., Beckers, F. (2003). Heart rate variability in athletes. Sports Medicine, 33(12), 889-919.

Bigger, J. T., Fleiss, J. L., Steinman, R. C., Rolnitzky, L. M., Kleiger, R. E., \& Rottman, J. N. (1992). Frequency domain measure of heart period variability and mortaliy after myocardial infarction. Circulation, 85, 164-171.

Caldeira, J. B., Soares, A. R., \& Amorim, V. O. (2009). Avaliação autonômica cardiovascular em indivíduos portadores de lesão medular completa submetidos ao teste de ortostatismo. Fisioterapia Brasil, 10(4), 251-56.

Cooke, W. H., Hoag, J. B., Crossman, A. A., Kuusela, T. A., Tahvanainen, K. U. O., \& Eckberg, D. L. (1999). Human responses to upright tilt: a window on central autonomic integration. Journal of Physiology, 517(2), 617-628.

Ditor, D. S., Kamath, M. V., MacDonald, M. J., Bugaresti, J., McCartney, N e Hicks, A. L. (2005). Reproducibility of heart rate variability and blood pressure variability in individuals with spinal cord injury. Clinical Autonomic Research, 15, 387-393.

Furlan, R., Gussetti, S., \& Crivellaro, W. (1990). Continuous 24 hour assessment of neural regulation of systemic arterial pressure and $\mathrm{RR}$ variabilities in ambulant subjects. Circulation, 81, 537-547.

Godoy, M. F., Takakura, I. T., Correa, P. R. (2005). Relevância da análise do comportamento dinâmico não-linear (Teoria do Caos) como elemento prognóstico de morbidade e mortalidade em pacientes submetidos à cirurgia de revascularização miocárdica. Arquivos de Ciências da Saúde, 12(4), 167-71.

Guyton, A. C., Hall, J. E. (2006). Tratado de fisiologia médica $\left(11^{\mathrm{a}}\right.$ ed). São Paulo: Elsevier Editora Ltda.

Guzzetti, S., Cogliati, C., Broggi, C., Carozzi, C., Caldiroli, D., Lombardi, F., \& Malliani, A. (1994). Influences of neural mechanisms on heart period and arterial pressure variabilities in quadriplegic patients. American Journal of Physiology, 266, H1112-H1120.

Inoue, .K, Miyake, S., Kumashiro, M., Ogata, H., \& Yoshimura, O. (1990). Power spectral analysis of heart rate variability in traumatic quadriplegic humans. American Journal of Physiology, 258, H1722-H1726.

Kleiger, R. E., Bosner, M. S., Rottman, J. N., \& Stein, P. K. (1993). Time-domain measurements of heart rate variability. Journal of Ambulatory Monitoring, 6, 1-18.

Koh, J., Brown, T. E., Beightol, L. A., Chang, H. Y., 
\& Eckberg, D. L. (1994). Human autonomic rhythms: vagal cardiac mechanisms in tetraplegic subjects. Journal of Physiology, 474 (3), 483-495.

Lopes, F. L., Pereira, F. M., Reboredo, M. M., Castro, T. M., Vianna, J. M., Novo Jr, J. M., \& Silva, L.P. (2007). Redução da variabilidade da frequência cardíaca em indivíduos de meia-idade e o efeito do treinamento de força. Revista Brasileira de Fisioterapia, São Carlos, 11(2),113-119.

Malik, M., Camm, A. J. (1996). The European society of cardiology the north American society of pacing and eletrophysiology: heart rate variability, standard of measurement, physiological interpretation and clinical use. Circulation, 93, 1043-1065.

Malik, M. (1995). Geometrical methods for heart rate variability assessment. In Malik, M., \& Camm, A. J, (Eds), Heart Rate Variability. Armonk, NY: Futura Publishing Company.

Malliane, A., Pagani, M., Lombardi, F., \& Cerruti, S. (1991). Cardiovascular neural regulation explored in the frequency domain. Circulation, $84,482-492$.

Moraes, R. S. F., \& Ferlin, E. L. (1992). Variabilidad de la frecuencia cardíaca: utilidad del análises espectral para evaluar el sistema nervioso autônomo. Revista Argentina de Cardiologia, 60, 77-80.

Mortara, A,. Rovere, L. A., \& Pantaleo, P. (1994). Relationship between baroreceptor sensitivity and sympathetic oscillations of heart rate variability in congestive heart failure. European Heart Journal, 15, 442.

Novais, L. D., Sakabe, D. I., Takahashi, A. C. M., Gongora, H., Taciro, C., Martins, L. E. B. (2004). Avaliação da variabilidade da frequência cardíaca em repouso de homens saudáveis sedentários e de hipertensos e coronariopatas em treinamento físico. Revista Brasileira de Fisioterapia, 8(3) 207-13.
Reis, A. F., Bastos, G. B., Mesquita, T. E., Romeo, M. J. L., \& Nóbrega, A. C. L. (1997). Disfunção parassimpática, variabilidade da frequência cardíaca e estimulação colinérgica após infarto agudo do miocárdio. Arquivos Brasileiros de Cardiologia, 68, 127.

Reis, M. S., Moreno, M. A., Sakabe, D. I., Catai, A. M. \& Silva, E. (2005). Análise da Modulação Autonômica da Frequência Cardíaca em Homens Sedentários e de Meia-idade. Fisioterapia em Movimento, Curitiba, 18(2) 11-18.

Ruha, A., Sallinen, S., \& Nissilä, S. (1997). A real-time microprocessor QRS detector system with a 1-ms timing accuracy for the measurement of ambulatory HRV. Transactions on Biomedical Engeneering, 44, 159-167.

Saul, J. P. (1990). Beat-to-beat variations of heart rate reflect modulation of cardiac autonomic outflow. News In Physiological Sciences, 5, 32-37.

Tsuji, H., Larson, M. G., \& Venditti, F. J. (1996). Impact of reduced heart rate variability on risk for cardiac events. Circulation, 94, 2850-2855.

Vanderlei, L. C. M., Pastre, C. M., Hoshi, R. A., Carvalho, T. D., Godoy, M. F. (2009). Nocões básicas de variabilidade da frequência cardíaca e sua aplicabilidade clínica. Revista Brasileira de Cirurgia Cardiovascular, 24(2), 205-217.

Wecht, J. M., De Meersman, R. E., Weir, J. P., Spungen, A. M., Bauman, W. A. (2003). Cardiac autonomic responses to progressive head-up tilt in individuals with paraplegia. Clinical Autonomic Research, 13, 433-438.

West, J. B. (2004). Pulmonary pathophysiology: the essentials. (7a ed.) Baltimore: Linppicott Willians and Wilkins.

(cc) EY-No Todo o conteúdo da revista Motricidade está licenciado sob a Creative Commons, exceto quando especificado em contrário e nos conteúdos retirados de outras fontes bibliográficas. 